\title{
Chronic active hepatitis in the type I polyglandular autoimmune syndrome
}

\author{
Theresa M. Michele, Jacqueline Fleckenstein, Anita R. Sgrignoli ${ }^{1}$ and \\ Paul J. Thuluvath \\ Division of Gastroenterology \& ' Pathology, The Johns Hopkins University Hospital, Baltimore, \\ MD 21205, USA
}

\begin{abstract}
Summary: Type I polyglandular autoimmune syndrome is characterized by the triad of hypoparathyroidism, Addison's disease and chronic mucocutaneous candidiasis. Chronic active hepatitis has been associated with this syndrome but its incidence and severity have not been well documented.

We describe a sibship of two patients with type I polyglandular autoimmune syndrome who presented with autoimmune chronic active hepatitis. The first patient presented to us with advanced disease and died despite an emergent liver transplant, while the second patient responded to steroids.

Autoimmune chronic active hepatitis, a major cause of mortality in this syndrome, can present without symptoms or physical signs of liver disease. We suggest periodic screening of liver enzymes in subjects with this syndrome.
\end{abstract}

\section{Introduction}

Type I autoimmune polyglandular syndrome, also known as autoimmune polyendocrinopathycandidiasis-ectodermal dystrophy (APECED), is a rare disorder defined by hypoparathyroidism, Addison's disease and chronic mucocutaneous candidiasis. The syndrome was first recognized by Whittaker in $1956 .{ }^{1}$ Other associated features include pernicious anaemia, diabetes mellitus, gonadal failure, autoimmune thyroid disease, vitiligo, alopecia, malabsorption, hypoplasia of dental enamel and nails, keratopathy and chronic active hepatitis. ${ }^{2-10}$ The syndrome usually occurs in childhood with the peak age of onset around 12 years. ${ }^{2,3,5,6}$ Studies suggest an autosomal recessive inheritance ${ }^{2,4,9}$ without HLA predisposition. ${ }^{2,3,7,9}$ Although liver disease has been reported in association with this syndrome, it is poorly described in the literature. We report a sibship of two patients with this syndrome, both of whom developed autoimmune hepatitis.

\section{Case reports}

Case 1

A 10 year old girl was transferred to our institution for evaluation of jaundice. She was the product of a

Correspondence: P.J. Thuluvath, M.B.B.S., M.D., M.R.C.P., Division of Gastroenterology, Blalock 935, Johns Hopkins Hospital, 600 N. Wolfe Street, Baltimore, MD 21205, USA.

Accepted: 29 July 1993 non-consanguineous marriage without family history of autoimmune disorders of any type except in her brother presented below. She was known to have type I polyglandular autoimmune syndrome since she was 3 years old. At the time of presentation she had hypoparathyroidism, Addison's disease and candidiasis involving nails and oral mucosa. The symptoms of liver disease started when she was 6 years old with lethargy, anorexia and jaundice that resolved spontaneously. She had multiple episodes of these symptoms during the next 4 years. At the time of admission to this hospital, her liver function tests showed aspartate aminotransferase (AST) 720 IU/l (normal 0-35 IU/l), alanine aminotransferase (ALT) $490 \mathrm{IU} / 1$ (normal 0-30 IU/1), alkaline phosphatase $300 \mathrm{IU} / 1$ (normal 30-120 IU/1, total bilirubin $600 \mu \mathrm{mol} / 1$, direct bilirubin $440 \mu \mathrm{mol} / 1$, albumin $36 \mathrm{~g} / \mathrm{l}$ and total protein $62 \mathrm{~g} / \mathrm{l}$.

Physical examination showed icterus, oral and nail candidiasis. There was no asterixis, hepatosplenomegaly or ascites. Hepatatis A IgM and hepatitis B s antigen and hepatatis B core IgM antibody, Coombs test and anti-nuclear antibody (ANF) were negative. Serum immunoglobulins (Ig) showed marginal increase in IgG (IgG $16.5 \mathrm{~g} / 1$, IgA $2.7 \mathrm{~g} / 1$, IgM $2.3 \mathrm{~g} / \mathrm{l}$ ). Serum ceruloplasmin and alpha-1-anti-trypsin were normal. Slit-lamp examination did not show Kayser-Flescher (KF) rings. Ultrasound examination of the liver was unremarkable. Liver histology showed a severe, somewhat patchy hepatitis with extensive areas of panlobular necrosis. The areas of panlobular nec- 
rosis showed considerable bile duct proliferation indicating a duration of at least several weeks. The histological changes were consistent with a severe chronic active hepatitis. There was an inflammatory infiltrate of lymphocytes and plasma cells, and areas of panlobular necrosis with considerable bile duct proliferation. She was started on prednisone $60 \mathrm{mg}$ daily on presentation, but she became encephalopathic and deteriorated rapidly over the next few days. She died despite an emergency liver transplant. The explant showed postnecrotic cirrhosis with little evidence of ongoing hepatitis (Figure 1).

\section{Case 2}

Six years after the death of the first patient, her 21 year old only sibling was admitted to our hospital for the management of symptomatic hypocalcaemia. He was diagnosed with type I polyglandular autoimmune syndrome when he presented at the age of seven with Addison's disease and hypoparathyroidism. He developed diabetes mellitus when he was 13 years old and candida oesophagitis 7 years later. He also suffered from intermittent diarrheoa and malabsorption.

Physical examination showed short stature, vitiligo, and positive Chovstek's and Trousseau's signs. Abdominal examination was unremarkable. Routine investigations revealed deranged liver biochemistry with AST $720 \mathrm{IU} / \mathrm{l}$, ALT 1,148 IU/l, alkaline phosphatase $363 \mathrm{IU} / 1$, total bilirubin $24 \mu \mathrm{mol} / 1$, direct bilirubin $10 \mu \mathrm{mol} / 11$, albumin $37 \mathrm{~g} / 1$ and total protein $68 \mathrm{~g} / \mathrm{l}$. Prothrombin time was 13.1 seconds (control 10.8-12.8 seconds). He had no risk factors for viral hepatitis, was not on any hepatotoxic drugs and did not consume excess alcohol. Serology for hepatitis A IgM antibody, hepatitis B s antigen, hepatitis B core IgM and hepatatis $C$ antibody were negative. Serum

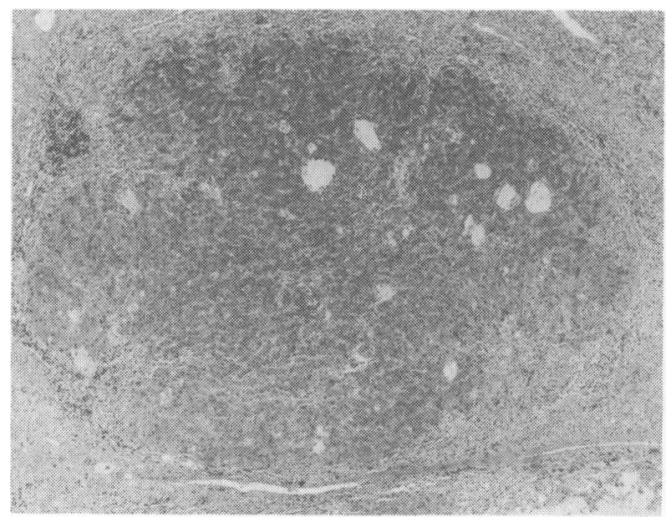

Figure 1 Case 1: liver explant approximately 2 months later showing cirrhosis. Remaining viable nodule with surrounding fibrosis and bile ductule proliferation, $\times 50$. ceruloplasmin, alpha-1-anti-trypsin and ferritin were normal. Anti-liver-kidney microsomal (antiLKM) antibody and anti-nuclear antibody (ANF) were negative; anti-mitochondrial antibody (AMA) and anti-smooth muscle antibody (ASMA) were weakly positive at $1: 40$ and $1: 20$, respectively. Immunoglobulins were raised (IgG $17 \mathrm{~g} / \mathrm{l}$, IgA $2.2 \mathrm{~g} / 1$ and $\operatorname{IgM} 3.6 \mathrm{~g} / \mathrm{l}$ ). Liver biopsy showed severe hepatitis with focal bridging and panlobular necrosis (Figure 2).

Although the histological findings were aetiologically non-specific, there was a prominent plasma cell component to the inflammatory infiltrate, suggestive of an autoimmune aetiology, and there was no clinical evidence for a viral- or drug-induced hepatitis. He was started on prednisolone $40 \mathrm{mg}$ daily. AST and ALT dropped to $40 \mathrm{IU} / 1$ and $100 \mathrm{IU} / 1$, respectively, within 2 weeks of therapy and returned to normal within 6 weeks. Prior to the steroid treatment the enzymes were slowly going up and the improvement was seen within 72 hours after initiating the treatment. A liver biopsy performed 3 months after treatment showed only mild chronic portal inflammation with mild expansion of some portal areas consistent with a minimally active hepatitis. There was significant histological improvement compared to his previous biopsy; this dramatic response to steroids lends further support for an autoimmune aetiology for the patient's hepatitis. ${ }^{11} \mathrm{He}$ has remained well on azathioprine $50 \mathrm{mg}$ daily and prednisolone $12.5 \mathrm{mg}$ daily. The serology for HBV and HCV have remained negative and his liver function tests have remained normal.

\section{Discussion}

The patients described here had the complete triad of candidiasis, hypoparathyroidism and adrenal

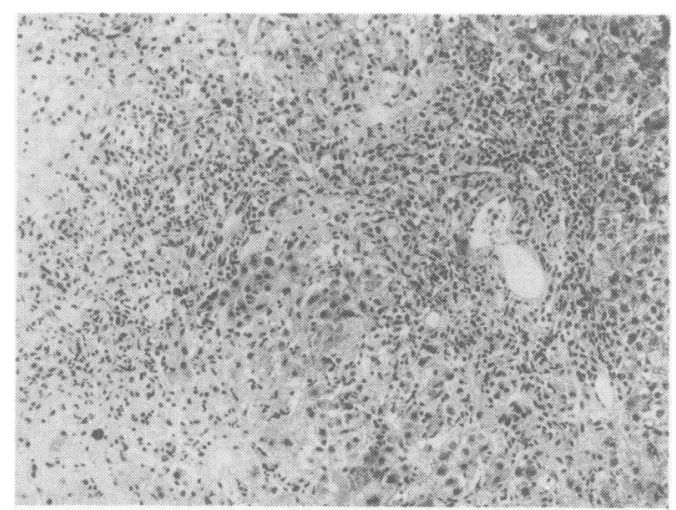

Figure 2 Case 2: Initial liver biopsy showing changes consistent with autoimmune hepatitis. Severe hepatitis with focal bridging and panlobular necrosis, $\times 160$. 
insufficiency. Case 1 had symptomatic episodes of liver disease for 4 years before she developed fulminant hepatitis. Case 2, on the contrary, was asymptomatic when he was found to have abnormal liver function tests. Both cases did not have all the characteristics of autoimmune liver disease. The immunoglobulins were only marginally raised in both cases and autoantibodies were negative. In the first case hepatitis $C$ was not excluded since the tests were unavailable at the time of her presentation. It is also possible that these patients may have had some form of viral hepatitis (? non-A, non-B, non-C hepatitis). A common infectious organism is unlikely since there was a gap of 6 years between the presentation of case 1 and 2 . The absence of risk factors for viral hepatitis and the rapid response to steroid also make viral hepatitis less likely. ${ }^{11}$ In case 1 , who required liver transplant, the inflammatory changes had settled down quite dramatically following 2 weeks of prednisone and in case 2 , the liver enzymes were rapidly increasing when prednisolone was started and the response was rapid and consistent. The liver biopsies of these patients had a significant number of plasma cells in the inflammatory component, although their serum immunoglobulins were not very high. Moreover, hypergammaglobulinemia need not always be present in autoimmune chronic active hepatitis, especially in children. ${ }^{12}$ On the basis of this evidence and due to its association with other autoimmune diseases, we believe that these two cases had autoimmune chronic active hepatitis.

Chronic liver disease has been reported before in association with type I polyglandular autoimmune syndrome, ${ }^{3,5,7}$ but not with type II or type III polyglandular syndrome. ${ }^{2,7,9}$ The initial observations of liver disease and its association with the type 1 syndrome were based on autopsy findings and isolated case reports. ${ }^{13-15}$ Cirrhosis and periportal fibrosis were incidental findings in many of these early reports. ${ }^{13,15}$ Before this syndrome was recognized as an autoimmune disease, and when markers for viral hepatitis were not available, it was suggested that this syndrome may be a sequel to 'hepatitis' despite the absence for clinical evidence of viral hepatitis. ${ }^{16}$ In a more recent study, Arulanantham et al. described a child who developed jaundice and ascites. ${ }^{9}$ This patient had high titres of smooth muscle antibody and high IgG levels. Liver biopsy showed plasma cell disruption of the limiting plate, rosette formation and areas of post-necrotic cirrhosis. The liver function of this patient improved with steroids and azathioprine, but the patient died as a result of viral pneumonia.

In a retrospective series of 41 patients reported by Neufield and Blizzard, six had chronic active hepatitis. ${ }^{7}$ The authors did not provide detailed information about the liver disease, but they suggested that it is life threatening and is the most difficulat organ-specific autoimmune disease to treat'. ${ }^{6}$ In a more recent review, Ahonen et al. reported follow-up data on 68 patients from Finland. ${ }^{5}$ Twelve patients had chronic hepatitis, in one of whom hepatitis was the initial presentation of the polyglandular autoimmune syndrome. Of the nine patients who died during the follow-up, two died due to fulminant hepatic failure; both had normal plasma aminotransferases prior to the onset of fatal hepatitis.

Lack of detailed information on the patients who had liver disease, from the published literature, make it difficult to draw any conclusions, but it appears that like the patients we have described, in this syndrome, the disease may be often subclinical and may have a fatal outcome if not treated early. It also appears that these patients do not exhibit all the characteristic features of autoimmune chronic active hepatitis. This observation emphasizes the importance of periodic screening of liver enzymes in subjects who are known to have this syndrome, especially as they may have no symptoms or physical signs of liver disease. Early diagnosis and intervention may prevent death due to liver failure in this rare syndrome.

In type I polyglandular syndrome, the more common disease components tend to present earlier on, with candidiasis and hypoparathyroidism being the usual presenting feature, closely followed by Addison's disease. ${ }^{5,7}$ Thus, it is unlikely that a polyendocrinopathy would be later detected in a subject who initially presents with autoimmune hepatitis, particularly if the subject is older than 20 years of age. In polyglandular autoimmunity, in general, antibody titres have not correlated well with either the presence of clinical disease or with eventual disease development. ${ }^{2}$

The exact mechanism of liver damage is this syndrome remains unclear. Hitherto no studies have demonstrated liver specific autoantibodies in type I polyglandular syndrome, although antibodies against thyroid, retro-orbital tissues, adrenal gland, gastric parietal cells, intrinsic factor, germ cells, pancreas, pituitary and smooth muscle have been identified. ${ }^{2,8}$ It has been suggested that defective T-cell function may explain the multiple clinical manifestations of the disease. ${ }^{7,9,17}$

Chronic active hepatitis is seen in $15-18 \%$ of patients with type I polyglandular autoimmune syndrome and it carries significant mortality. ${ }^{2,5,7,9,15}$ Periodic screening, a high index of suspicion, and early diagnosis and treatment will reduce mortality from liver disease in this syndrome. 


\section{References}

1. Whitaker, J., Landing, B.H., Esselborn, V.M. \& Williams, R.R. The syndrome of familial juvenile hypoadrenocorticism, hypoparathyroidism and superficial moniliasis. J Clin Endocrinol Metab 1956, 16: 1374-1387.

2. Trence, D.L., Morley, J.E. \& Handwerger, B.S. Polyglandular autoimmune syndromes. Am J Med 1984, 77: 107-116.

3. Brun, J.M. Juvenile auto-immune polyendocrinolopathy. Hormone Res 1982, 16: 308-316.

4. Ahonen, P. Autoimmune polyendocrinopathy-candidiasisectodermal dystrophy (APECED): autosomal recessive inheritance. Clin Genetics 1985, 27: 535-542.

5. Ahonen, P., Myllarniemi, S., Sipila, I. \& Perheentupa, J. Clinical variation of autoimmune polyendocrinopathycandidiasis-ectodermal dystrophy (APECED) in a series of 68 patients. $N$ Engl J Med 1990, 322: 1829-1836.

6. Neufield, M. \& Blizzard, R.M. Polyglandular autoimmune disease. In: Pincherra, A., Doniach, D., Fenzi, G.F. \& Baschieri, L. (eds) Autoimmune Aspects of Endocrine Disorders. Proceedings of the Serona Symposia, Vol. 33. Academic Press, New York, 1980, pp. 357-365.

7. Neufield, M., Maclaren, N.K. \& Blizzard, R.M. Two types of auto-immune Addison's disease associated with different polyglandular autoimmune (PGA) syndromes. Medicine (Baltimore) 1981, 60: 355-362.

8. Barkan, A.L., Kelch, R.P. \& Marshall, J.C. Isolated gonadotrope failure in the polyglandular autoimmune syndrome. $N$ Engl J Med 1985, 312: 1535-1540.

9. Arulanantham, K., Dwyer, J.M. \& Genel, M. Evidence for defective immunoregulation in the syndrome of familial candidiasis endocrinopathy. $N$ Engl $J$ Med 1979, 300: 164-168.
10. Friedman, T.C., Thomas, P.M., Fleisher, T.A. et al. Frequent occurrence of asplenism and cholelithiasis in patients with autoimmune polyglandular disease Type 1. Am J Med 1991, 91: 625-630.

11. Schaffner, F. Autoimmune chronic active hepatitis; three decades of progress. In: Popper, H. \& Schaffner, F. (eds) Progress in Liver Disease, Vol. 8. Orlando, Grune \& Stratton, 1986 , p. 485

12. Maggiore, G., Porta, G., Bernard, O. et al. Autoimmune hepatitis with initial presentation as acute hepatic failure in young children. $J$ Pediatr 1990, 116: 280-282.

13. Craig, J.M., Schiff, L.H. \& Boone, J.E. Chronic moniliasis associated with Addison's disease. Am J Dis Child 1955, 89: 669-683.

14. Perlmutter, M., Ellson, R.R., Norsa, L. \& Kantrowitz, A.R. Idiopathic hypoparathyroidism and Addison's disease. Am J Med 1956, 21: 634-643.

15. Williams, E. \& Wood, C. The syndrome of hypoparathyroidism and steatorrhoea. Arch Dis Childhood 1959, 34: 302-306.

16. Kunin, A.S., MacKay, B.R., Burns, S.L. \& Halberstam, M.J. The syndrome of hypoparathyroidism and adrenocortical insufficiency, a possible sequel of hepatitis. Am J Med 1963 , 34: 856-866.

17. Kaffe, S., Petigrow, C.S., Cahill, L.T. et al. Variable cellmediated immune defects in a family with "candida endocrinopathy syndrome.' Clin Exp Immunol 1975, 20: 397-408. 